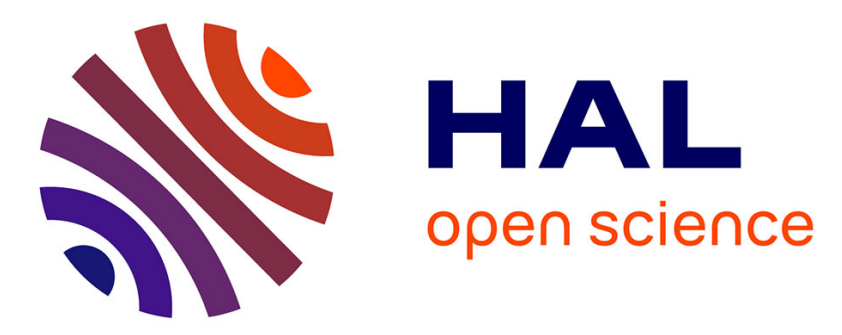

\title{
Freezing and Melting of Silver Nanoparticles on Silica Substrate Using a Simple Interatomic Potential for Ag-SiO 2 Interaction on the Basis of ab Initio Calculations and Experimental Data
}

\author{
Alain Cabrel Ngandjong, C. Mottet, Joël Puibasset
}

\section{To cite this version:}

Alain Cabrel Ngandjong, C. Mottet, Joël Puibasset. Freezing and Melting of Silver Nanoparticles on Silica Substrate Using a Simple Interatomic Potential for Ag-SiO 2 Interaction on the Basis of ab Initio Calculations and Experimental Data. Journal of Physical Chemistry C, 2017, 121 (6), pp.3615 - 3622. 10.1021/acs.jpcc.6b12084 . hal-01720749v2

\author{
HAL Id: hal-01720749 \\ https://hal.science/hal-01720749v2
}

Submitted on 31 Jan 2022

HAL is a multi-disciplinary open access archive for the deposit and dissemination of scientific research documents, whether they are published or not. The documents may come from teaching and research institutions in France or abroad, or from public or private research centers.
L'archive ouverte pluridisciplinaire HAL, est destinée au dépôt et à la diffusion de documents scientifiques de niveau recherche, publiés ou non, émanant des établissements d'enseignement et de recherche français ou étrangers, des laboratoires publics ou privés. 


\title{
Freezing and Melting of Silver Nanoparticles on Silica Substrate Using a Simple Interatomic Potential for $\mathrm{Ag}-\mathrm{SiO}_{2}$ Interaction on the Basis of $a b$ Initio Calculations and Experimental Data
}

\author{
A. C. Ngandjong, ${ }^{\dagger}$ C. Mottet, ${ }^{\ddagger}$ and J. Puibasset* ${ }^{*} \dagger$ \\ ${ }^{\dagger}$ Interfaces, Confinement, Matériaux et Nanostructures (ICMN), UMR 7374, CNRS et Université d'Orléans, 1b rue de la Férollerie, \\ 45071 Orléans cedex 02, France \\ ${ }^{\ddagger}$ Aix-Marseille Université, CNRS, CINaM UMR7325, Campus de Luminy, case 913, 13288 Marseille, cedex 9, France
}

ABSTRACT: We have devised a $\mathrm{Ag}-\mathrm{SiO}_{2}$ potential for Monte Carlo and molecular dynamics studies of systems comprising silver nanoparticles in contact with silica surfaces. The potential is determined based on density functional theory (DFT) calculations on $\beta$-cristobalite, a silica crystal, as well as theoretical and experimental data on $\alpha$-quartz and amorphous silica found in literature. The interactions between $\mathrm{Ag}$ and silica species are fitted with the simple Lennard-Jones $(12,6)$ potential with parameters $\sigma_{\mathrm{Ag}-\mathrm{O}}=0.278 \mathrm{~nm}, \sigma_{\mathrm{Ag}-\mathrm{Si}}=0.329 \mathrm{~nm}, \varepsilon_{\mathrm{Ag}-\mathrm{O}}=$ $0.012 \mathrm{eV}$, and $\varepsilon_{\mathrm{Ag}-\mathrm{Si}}=0.002 \mathrm{eV}$. The nature of the substrate is taken into account at a phenomenological level as an extra multiplicative factor $\eta$ for the total interaction between silver and silica: for $\alpha$-quartz $\eta^{\text {quartz }}=1$, for hydroxylated $\beta$-cristobalite $\eta^{\text {crist }}=5.0$, and for amorphous silica $\eta^{\text {amorph }}=2.5$. This potential is thought to be useful to investigate the effect of the silica support on the structure and morphology of deposited silver nanoparticles. As an example, we study the melting and freezing of supported nanoparticles. This potential could also possibly be extended to other metals or support.

\section{INTRODUCTION}

Nanoparticles are model catalysts widely studied by various techniques. ${ }^{1-4}$ In most cases, they are deposited on a substrate (they are either grown directly on the surface or initially produced in a beam before being deposited). ${ }^{1-9}$ Generally an oxide support is chosen in order to minimize the interaction and thus keep a three-dimensional morphology closest as possible to the ideal situation free of interaction. Crystalline oxides, like $\mathrm{MgO}$, are known to significantly influence the structure and morphology of the deposited nanoparticle. ${ }^{7-19}$ This is why amorphous substrates (silica or carbon) are generally preferred, but even in that case they may have nonnegligible effects, as suggested by experiments. ${ }^{20,21}$ Understanding the interaction of metallic nanoparticles with amorphous substrates is thus an important issue, which is actually not so well documented because of the intrinsic complexity of amorphous systems. In particular, the chemistry and structure of silica surfaces ${ }^{22-24}$ exhibit a large variability, including various degree of hydroxylation that can be modeled with empirical potentials or $a b$ initio density functional theory (DFT). ${ }^{25-36}$

Silver nanoparticles present interesting optical properties leading to many applications in plasmonics, surface-enhanced spectroscopies or biosensors. ${ }^{37-40}$ These properties highly depend on the shape and size of the nanoparticles and a better theoretical understanding of the influence of the amorphous silica support on the structural stability of silver nanoparticles would be helpful. However, for large systems, DFT calculations become intractable and semiempirical potentials for Monte Carlo or molecular dynamics simulations have proven to be useful. Such potentials have already been devised to describe silica $^{25-28}$ on one side, and transition metals like silver on the other side. ${ }^{13,41,42}$ However, data regarding the silver-silica interaction are scarce. Experiments and theory show that nonreactive metals interact weakly with silica. X-ray photoemission spectroscopy shows that the electronic structure of a silver atom implanted in silica is close to that of the gas phase free atom. ${ }^{43}$ Sessile drop measurements performed by Eusthatopoulos and co-workers ${ }^{44-46}$ give a wetting angle of $140^{\circ}$ for the $\mathrm{Ag}-\mathrm{SiO}_{2}$ system, typical of weak van der Waals like interactions. $A b$ initio calculations of silver atoms deposited on a silica crystal ( $\alpha$-quartz) also show that the interactions are weak. ${ }^{47}$ An empirical van der Waals like potential can thus be determined to reproduce the $\mathrm{Ag}-\mathrm{SiO}_{2}$ interactions. The objective of our work is 2-fold: (i) complete these data with complementary $a b$ initio calculations, and (ii) determine an empirical $\mathrm{Ag}-\mathrm{SiO}_{2}$ potential based on all available data.

(i) In order to complete the DFT results obtained by Vakula et al. $^{47}$ on $\alpha$-quartz, we have considered another silica crystal: the $\beta$-cristobalite. This substrate differs significantly from the quartz in terms of structure and density.

Received: December 1, 2016

Revised: January 18, 2017

Published: January 19, 2017 
Furthermore, quartz exhibits low energy surfaces with the same stoichiometry as the bulk $\left(\mathrm{SiO}_{2}\right)$, while cristobalite faces are highly reactive with water vapor and essentially exhibit hydroxylated surfaces (presence of hydroxyl groups -OH). The degree of hydroxylation depends on the temperature and the water vapor pressure, and may vary between no hydroxyls up to $7 \mathrm{OH} / \mathrm{nm}^{2}$ at full saturation. $^{22-24}$ Comparing quartz with cristobalite is thus an interesting route to understand the effect of structure and surface chemistry (hydroxylation) which is known to play an important role in metal-silica interactions, as shown for instance by DFT calculations on similar metal- $\mathrm{SiO}_{2}$ systems. ${ }^{36,48}$ Furthermore, the density of $\beta$-cristobalite is close to that of amorphous silica, and the results could thus be transferable to that important material used in many deposition experiments (supports made of oxidized silicon wafers). Note that direct calculations of $\mathrm{Ag}$ interacting with amorphous silica could be possible in near future. ${ }^{33-36}$ For the present study we focus on the simple periodic cristobalite structure, with various surface hydroxyl densities.

(ii) The second objective is to use all available data (DFT calculations and experimental results) to determine a simple empirical $\mathrm{Ag}-\mathrm{SiO}_{2}$ potential. Since calculations and experiments show that the interactions are weak and most probably essentially van der Waals like, ${ }^{43-46}$ the general form of the metal-silica species interactions is chosen to be the Lennard-Jones $(12,6)$ :

$$
\mathrm{V}_{\mathrm{A}-\mathrm{B}}(r)=4 \eta^{\text {surf }} \varepsilon_{\mathrm{A}-\mathrm{B}}\left[\left(\frac{\sigma_{\mathrm{A}-\mathrm{B}}}{r}\right)^{12}-\left(\frac{\sigma_{\mathrm{A}-\mathrm{B}}}{r}\right)^{6}\right]
$$

Two parameters (an energy $\varepsilon$ and a distance $\sigma$ ) depend on the nature of the interacting species $A-B$, while $\eta^{\text {surf }}$ is a global dimensionless parameter that depends only on the nature of the silica substrate. Ignoring this parameter would implicitly rely on the hypothesis that the interactions barely depend on the silica surface structure (quartz, cristobalite or amorphous) and chemistry (hydroxylation), which is not supported by our calculations. The quartz surface being stoichiometric and nonhydroxylated (pure $\mathrm{SiO}_{2}$ ), it is used as the reference: $\eta^{\text {quartz }}$ $=1$. In other words, $\eta^{\text {surf }}$ measures the intensity of the $\mathrm{Ag}-\mathrm{SiO}_{2}$ interaction relative to the $\alpha$-quartz surface. When the silica is hydroxylated, a rigorous treatment would consider the interactions between metal and hydrogen species, and should distinguish between surface oxygen (involved in hydroxyl groups $\mathrm{Si}-\mathrm{O}-\mathrm{H})$ from bulk oxygen species $(\mathrm{Si}-\mathrm{O}-\mathrm{Si})$. We made the choice to keep the number of free parameters as small as possible: the $\mathrm{O}$ species are not distinguished, and the effect of the hydrogen atoms is included in the $\mathrm{Ag}-\mathrm{O}$ interactions; i.e., the hydroxyl groups are treated as point-like, and the modulation in the intensity of the interactions due to hydroxylation is taken into account at a phenomenological

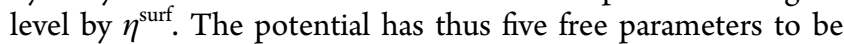
determined ( $\left.\eta^{\text {surf }}, \sigma_{\mathrm{Ag}-\mathrm{O}}, \sigma_{\mathrm{Ag}-\mathrm{Si}}, \varepsilon_{\mathrm{Ag}-\mathrm{O}}, \varepsilon_{\mathrm{Ag}-\mathrm{Si}}\right)$.

The paper is organized as follows: section 2 presents the general route to determine the $\mathrm{Ag}$-silica potential as well as the DFT and Monte Carlo methods used in this work; section 3 gives the results for the adsorption and adhesion energy on cristobalite and the $\mathrm{Ag}$-silica potential deduced from all available data, followed by a study of the melting and freezing of nanoparticles on silica using Monte Carlo simulations in the canonical ensemble.

\section{METHODS}

2.1. General Procedure to Determine the $\mathrm{Ag}-\mathrm{SiO}_{2}$ Potentials. The reference system Ag-quartz is first considered, since in that case $\eta^{\text {quartz }}=1$ and only four free parameters have to be determined. This can be done based on the DFT calculations by Vakula and co-workers ${ }^{47}$ because the surface of quartz exhibits two attractive sites for isolated silver atoms, giving enough geometric and energetic information to determine the Lennard-Jones parameters.

The parameter $\eta^{\text {crist }}$ for cristobalite is then determined from DFT calculations of the adsorption (or adhesion) energy of an isolated atom or one to four layers of silver deposited on the surface. The adhesion energy $E_{\mathrm{ad}}$ is defined by

$$
E_{\mathrm{ad}}=E\left(\mathrm{Ag}+\mathrm{SiO}_{2}\right)-E\left(\mathrm{SiO}_{2}\right)-E(\mathrm{Ag})
$$

were $E(\mathrm{Ag})$ is the energy of silver, $E\left(\mathrm{SiO}_{2}\right)$ the energy of the silica slab and $E\left(\mathrm{Ag}+\mathrm{SiO}_{2}\right)$ the energy of the complete system $\left(\mathrm{Ag}+\mathrm{SiO}_{2}\right)$. When an assembly of $\mathrm{Ag}$ atoms is considered, this quantity is divided by the number of $\mathrm{Ag}$ atoms at the interface. The parameter $\eta^{\text {crist }}$ is determined by requiring that the empirical potential (eq 1 , with $\varepsilon$ and $\sigma$ previously determined on quartz) gives the same adhesion energy as obtained from DFT. Note that the only free parameter is $\eta^{\text {crist }}$, and that the $\mathrm{Ag}-\mathrm{SiO}_{2}$ interaction being weak the adhesion energy is essentially proportional to $\eta^{\text {crist }}$. More details on the DFT method are given below.

The parameter $\eta^{\text {amorph }}$ for amorphous silica is determined based on wetting angle measurements. The procedure consists in reproducing numerically the experiment (by Monte Carlo simulations $)^{49}$ with a nanodroplet deposited on an amorphous silica surface. This method uses the $\mathrm{Ag}-\mathrm{SiO}_{2}$ potential eq 1 but also semiempirical potentials to model the metal-metal and the silica species interactions (see below).

2.2. DFT Calculations. The DFT calculations are performed to determine the physical quantities of interest (geometry and energy) on small systems comprising a silica crystal surface (substrate) and a single silver atom or one to four layers. We use the Vienna $a b$ initio simulation package (VASP) code and the projector augmented wave (PAW) $)^{50,51}$ interaction potential between the electrons and the ions. The exchange and correlation functional is the generalized gradient approximation (GGA-PBE). ${ }^{52}$ The Brillouin zone integration is performed with the Monkhorst-Pack scheme with $k$-point mesh comprising 10 subdivisions along each reciprocal lattice vector and only one subdivision in the direction perpendicular to the surface. The energy cutoff is taken equal to $450 \mathrm{eV}$ for each type of atom. We have also considered the inclusion of the van der Waals dispersive interaction in the DFT calculations using the Grimme method called "DFT-D2" as implemented in the VASP code. ${ }^{53}$ The dispersive correction in the Grimme model takes into account polarization interactions as a sum of terms $\mathrm{C}_{i j} / \mathrm{r}_{i j}{ }^{6}$ where the parameters $\mathrm{C}_{i j}$ are computed using the following combination rules: $C_{i j}{ }^{2}=C_{i i} C_{j j}$, and the $C_{i i}$ values are tabulated for each element. These dispersion terms have proven to be essential in some systems. ${ }^{54}$

A bloc of bulk $\beta$-cristobalite is optimized with periodic boundary conditions in the three directions. Its lattice parameter is found to be equal to $0.746 \mathrm{~nm}$ (to be compared with the experimental value of $0.716 \mathrm{~nm}$ ). The surface of cristobalite is made by introducing a void in the simulation box parallel to the (001) plane. The box initially comprises $8 \mathrm{Si}$ atoms and $16 \mathrm{O} ; 4$ extra $\mathrm{O}$ are added so that both faces are 
saturated with $\mathrm{O}$ and identical. After optimization, the surface oxygen species form dimers (see Figure 1). The obtained

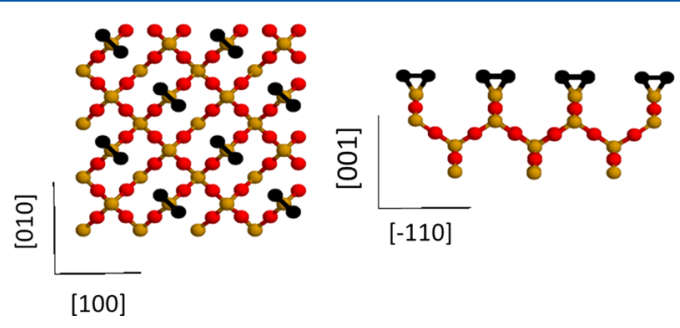

Figure 1. Top (left) and side (right) views of the nonhydroxylated (001) $\beta$-cristobalite surface after relaxation of atomic positions. Key: yellow, $\mathrm{Si}$; red, bulk $\mathrm{O}$; black, surface $\mathrm{O}$, which form dimers. For illustration, the cell is duplicated twice in both directions parallel to the surface.

surface is nonhydroxylated, and is useful for comparisons with the pure quartz, but is probably not realistic. Experimental study under vacuum show that the mean density of hydroxyl groups on amorphous silica surface is $4.6 \mathrm{OH} / \mathrm{nm}^{2}$ for temperature between 180 and $200{ }^{\circ} \mathrm{C}$, and $3.5 \mathrm{OH} / \mathrm{nm}^{2}$ at $300{ }^{\circ} \mathrm{C} .{ }^{22,23}$ These conditions correspond to deposition experiments on silicon wafers. In order to mimic this hydroxylated amorphous silica surface, it is possible to consider various surface orientations of hydroxylated $\beta$-cristobalite, in particular (001) and (111), with adjusted proportions. ${ }^{55-57}$ In this work we focus on the (001) surface of $\beta$-cristobalite, made in an initial slab of $8 \mathrm{Si}$ atoms and $16 \mathrm{O}$ atoms. The surface is thus fully hydroxylated by saturating $\mathrm{Si}$ with $\mathrm{OH}$ groups and $\mathrm{O}$ with $\mathrm{H}$ atoms. This leads to add $4 \mathrm{H}_{2} \mathrm{O}$ molecules to our slab. The corresponding surface density of hydroxyl groups is 7.2 $\mathrm{OH} / \mathrm{nm}^{2}$. We also considered a partially hydroxylated surface corresponding to coverage of $3.6 \mathrm{OH} / \mathrm{nm}^{2}$, closer to experimental measurements, by removing $2 \mathrm{H}$ and $2 \mathrm{OH}$ (silanization) on the upper face where silver will be deposited. Both hydroxylated surfaces are shown in Figure 2. Then we considered three different metal deposits: one single $\mathrm{Ag}$ adatom (per $\mathrm{SiO}_{2}$ mesh which means with a distance of $0.746 \mathrm{~nm}$ between the $\mathrm{Ag}$ atoms), one (001) monolayer (i.e., $8 \mathrm{Ag}$ atoms
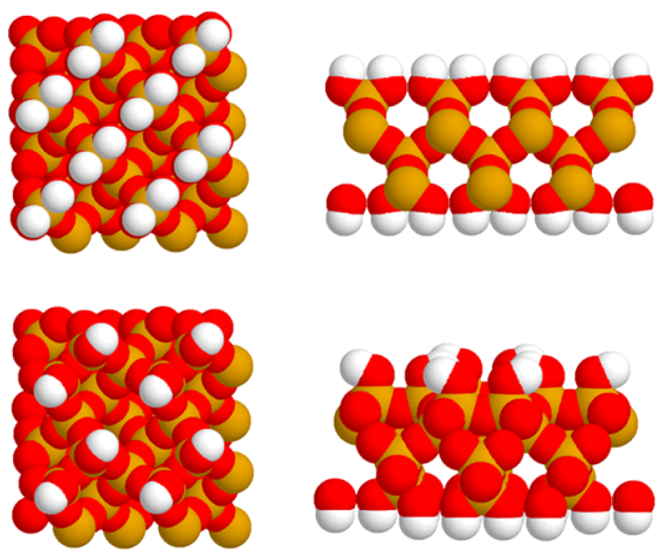

Figure 2. Relaxed (001) $\beta$-cristobalite surfaces: (a) full hydroxylation on both faces $\left(7.2 \mathrm{OH} / \mathrm{nm}^{2}\right)$; (b) partial hydroxylation of the upper face $\left(3.6 \mathrm{OH} / \mathrm{nm}^{2}\right)$. The left-hand panels are top views, and the righthand panels are side views along the diagonal direction. For illustration, the cell is duplicated twice in both directions parallel to the surface. per $\mathrm{SiO}_{2}$ mesh) and four (001) layers (i.e., $32 \mathrm{Ag}$ atoms per $\mathrm{SiO}_{2}$ mesh). The silver deposit can be deformed by the interaction with the silica substrate in order to minimize the total energy of the system. As a consequence, we consider the adsorption energy as the energy difference between the optimized structure of the $\mathrm{Ag} / \mathrm{SiO}_{2}-\mathrm{H}$ system and the $\mathrm{Ag}$ deposited with the same structure it has when it is deposited.

2.3. Monte Carlo Simulations. Monte Carlo simulations are used to mimic a wetting angle measurement experiment. The objective is to determine the silver-silica interaction intensity which reproduces the observed wetting angle. The metal-silica interaction is described by eq 1 , with $\varepsilon$ and $\sigma$ previously determined on quartz ( $\eta^{\text {amorph }}$ is the only free parameter of the simulation). The metal-metal interactions are modeled by the semiempirical many-body potential derived from the tight binding scheme in the second moment approximation (TBSMA). ${ }^{41}$ The energy at site " $n$ " writes in two terms. An attractive many-body term coming from the band energy in the form:

$$
E_{n}^{\mathrm{b}}=-\sqrt{\sum_{r_{n m}<r_{\mathrm{c}}}^{m \neq n} \xi^{2} \exp \left[-2 q\left(\frac{r_{\mathrm{nm}}}{r_{0}}-1\right)\right]}
$$

where " $r_{n m}$ " is the interatomic distance between atom at site " $n$ " and its neighbor at site " $m$ ", with $r_{n m}<\mathrm{r}_{\mathcal{c}}$ the cutoff distance equivalent to the distance of the second neighbors in bulk Ag, where $r_{0}$ is the first-neighbor distance. The second term is a core-repulsion term of the Born-Mayer type:

$$
E_{n}^{\mathrm{r}}=\sum_{r_{n m}<r_{\mathrm{c}}}^{m \neq n} A \exp \left[-p\left(\frac{r_{n m}}{r_{0}}-1\right)\right]
$$

where $p=10.790, q=3.190, A=0.10433 \mathrm{eV}$, and $\xi=1.19402$ $\mathrm{eV}$ are four parameters fitted to the bulk cohesive energy, lattice parameter and elastic constants. ${ }^{13,42}$ After the second neighbors, the potential is continuously prolonged up to zero by a fifth order polynomial form to cancel the interaction after the third neighbor distance.

The silica substrate is modeled with the m-TTAM potential. $^{25,27}$ The TTAM ${ }^{25}$ potential developed by Tsuneyuki, Tsukada, Aoki, and Matsui is based on the idea that the local tetrahedral structures present in silica can be reproduced with two-body spherically symmetric potentials acting on $\mathrm{Si}^{4+}$ and $\mathrm{O}^{2-}$ ions. The analytical form of the potential if of BornHuggins-Mayer type, including electrostatics, soft core and dispersion forces. This potential is very simple to implement in a molecular dynamics simulation, and has proven to be accurate enough to describe the various crystalline polymorphs of silica. At high temperature, the high kinetic energy of the atoms allows them to occasionally override the atom-atom repulsion barrier at very short-range where the dispersive term spuriously dominates. Guissani and Guillot ${ }^{27}$ cured this problem by adding a $r^{-18}$ repulsive term, and thus improved the potential at high temperature (up to $5000 \mathrm{~K}$ ). This potential is used to elaborate our amorphous silica support, as follows: a silica melt initially at $2000 \mathrm{~K}$ is cooled down to $300 \mathrm{~K}$. This produces a solid which is cut to form a slab. It is then relaxed at $300 \mathrm{~K}$, leading to a flat amorphous surface. This surface is kept rigid when the metallic droplet is introduced to mimic the wetting angle measurement experiment, a reasonable approximation considering the strong rigidity of silica compared with the weak metal-silica interaction. A typical configuration is shown in Figure 3. 


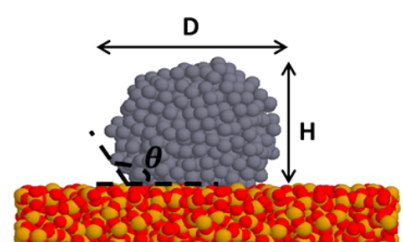

Figure 3. Snapshot of the Monte Carlo model for the wetting angle experiment, showing the silver nanodroplet (gray atoms) deposited on the amorphous silica support (yellow, $\mathrm{Si}$; red, O). $\theta$ is the wetting angle, and $\mathrm{H} / \mathrm{D}$ measures the aspect ratio of the droplet.

\section{RESULTS AND DISCUSSION}

3.1. Ag-Quartz Potential. The free parameters of eq 1 are determined from the DFT calculations by Vakula and coworkers on quartz. ${ }^{47}$ They have shown that the (001) surface of quartz, which undergoes a $(1 \times 1)$ reconstruction, exhibits two attractive sites for isolated silver atoms: above the center of a six-membered ring, and above one $\mathrm{Si}$ atom which is the meeting point of three six-membered rings. For each site, they have determined the adsorption energy and the equilibrium distances to neighboring atoms. For the first site, the distance from the silver atom to the center of the ring is $0.3201 \mathrm{~nm}$ and the adsorption energy is $-0.08 \mathrm{eV}$; for the second attractive site, the $\mathrm{Ag}-\mathrm{Si}$ distance is $0.3703 \mathrm{~nm}$ and the adsorption energy is $-0.06 \mathrm{eV}$. To determine the four parameters $\sigma_{\mathrm{Ag}-\mathrm{O}}$, $\sigma_{\mathrm{Ag}-\mathrm{Si}}, \varepsilon_{\mathrm{Ag}-\mathrm{O}}$, and $\varepsilon_{\mathrm{Ag}-\mathrm{Si}}\left(\eta^{\text {quartz }}=1\right)$, we require that the $\mathrm{LJ}$ potential eq 1 reproduces the same equilibrium positions and energies for a free isolated $\mathrm{Ag}$ atom above the quartz surface, which is kept immobile during the optimization. More specifically, the two positions (height) of the attractive sites uniquely determine $\sigma_{\mathrm{Ag}-\mathrm{O}}$ and $\sigma_{\mathrm{Ag}-\mathrm{Si}}$, and the two adsorption energies uniquely determine $\varepsilon_{\mathrm{Ag}-\mathrm{O}}$, and $\varepsilon_{\mathrm{Ag}-\mathrm{Si}}$. The results are given in Table 1 . As can be seen, the interactions are weak and dominated by the $\mathrm{O}$ species, as expected from their high polarizability.

Table 1. Lennard-Jones Parameters for the $\mathrm{Ag}-\mathrm{SiO}_{2}$ Interaction Potential (Eq 1) Determined from the Two Equilibrium Positions of a Single Ag Atom on the $\alpha$-Quartz (001) Surface (from Ref 47)

\begin{tabular}{lll} 
& $\mathrm{Ag}-\mathrm{O}$ & $\mathrm{Ag}-\mathrm{Si}$ \\
$\sigma(\AA)$ & 2.78 & 3.29 \\
$\varepsilon(\mathrm{eV})$ & 0.012 & 0.002 \\
\hline
\end{tabular}

3.2. Ag-Cristobalite Potential. 3.2.1. Non-Hydroxylated Surface. We first introduce an isolated $\mathrm{Ag}$ atom above the surface of the nonhydroxylated cristobalite to calculate its adsorption energy after optimization using DFT. Because of periodic boundary conditions parallel to the surface, one Ag per $\mathrm{SiO}_{2}$ mesh means a distance of $0.746 \mathrm{~nm}$ between the $\mathrm{Ag}$ atoms. We obtain high adsorption energy of approximately -5 $\mathrm{eV}$, in correlation with the observation that the silver atom maximizes the number of bonds with the surface oxygen atoms (see Table 2). We have also considered the case of Ag layers interacting with the surface to reproduce the situation of a deposited nanoparticle which generally exhibits flat facets. Because of mismatch between the silver layer and the cristobalite (001) face, the silver layer is contracted (9\%) to fit within the simulation box. After optimization of the system, we obtain a corrugated silver layer, where $\mathrm{Ag}-\mathrm{Ag}$ distances can recover their bulk value $(0.29 \mathrm{~nm})$. The corresponding
Table 2. DFT/DFT-D2 Calculations of the Adsorption Energy of an Isolated Ag Atom (eV) or Adhesion Energy of $\mathrm{Ag}$ Layers (eV per Ag Atom at the Interface) Deposited on the (001) Face of $\boldsymbol{\beta}$-Cristobalite with Various Degrees of Hydroxylation

\begin{tabular}{lccc} 
& nonhydroxylated & $3.6 \mathrm{OH} / \mathrm{nm}^{2}$ & $7.2 \mathrm{OH} / \mathrm{nm}^{2}$ \\
$1 \mathrm{Ag}$ atom $(\mathrm{eV})$ & $-4.8 /-5.34$ & $-0.16 /-0.36$ & $-0.05 /-0.14$ \\
$1 \mathrm{Ag}$ layer $(\mathrm{eV} / \mathrm{at})$ & $-0.69 /-1.04$ & $-0.04 /-0.12$ & $+0.01 /-0.15$ \\
$4 \mathrm{Ag}$ layers $(\mathrm{eV} /$ & $-0.57 /-0.85$ & $-0.04 /-0.19$ & $-0.02 /-0.16$ \\
at) & & & \\
\hline
\end{tabular}

adhesion energy is found to be $-0.69 \mathrm{eV}$ per surface $\mathrm{Ag}$ atoms, and $-1.04 \mathrm{eV} /$ at when the dispersion terms are taken into account with DFT-D2 (see Table 2). These values are significantly higher than that found for an isolated atom (ca. -5 $\mathrm{eV}$ ), showing the large influence of the silver-silver interaction on the silver-silica adhesion energy. We have also considered a slab made of four $\mathrm{Ag}$ layers: the corresponding adhesion energies are found to be $-0.57 \mathrm{eV}$ and $-0.85 \mathrm{eV}$ (with DFTD2) per surface atom at the $\mathrm{Ag}-\mathrm{SiO}_{2}$ interface (see Table 2). The strength of the $\mathrm{Ag}$-silica interaction thus slightly decreases when the coordination number of the $\mathrm{Ag}$ species at the interface increases (coordination 4 for one layer, and 8 for a thick slab).

The adhesion energies found on nonhydroxylated cristobalite are more than 1 order of magnitude larger than that found by Vakula and co-workers on quartz. Both substrates are pure $\mathrm{SiO}_{2}$ (nonhydroxylated), but they differ in bulk and surface structures. In particular the cristobalite surface (001) is polar $^{58}$ which may explain the strong interaction with silver atoms. The determination of the $\eta$ parameter of the silver-silica potential eq 1 to fit this large adhesion energy would be meaningless since the interactions are far from being van der Waals. Furthermore, the nonhydroxylated surface of cristobalite is probably not realistic since it is highly reactive, and its hydroxylation is expected to play an important role since it influences the polarity of the surface.

3.2.2. Hydroxylated Surface. The surface of freshly cut cristobalite is highly polar and reactive. In particular, exposition to ambient air leads to water dissociation at the surface and the formation of silanols. This is why we now consider partially and fully hydroxylated surfaces $\left(3.6 \mathrm{OH} / \mathrm{nm}^{2}\right.$ and $7.2 \mathrm{OH} / \mathrm{nm}^{2}$; see details in previous section). One silver atom or a full layer is then introduced above the surface and the geometry optimized. Without the dispersion contributions, the adsorption energy for an isolated atom is $-0.16 \mathrm{eV}$ for the partially hydroxylated surface and $-0.05 \mathrm{eV}$ for the fully hydroxylated surface. For a silver layer, one gets $-0.04 \mathrm{eV} /$ at and $+0.01 \mathrm{eV} /$ at respectively (see Table 2). By comparison with the nonhydroxylated surface, the interaction is significantly attenuated, as expected since the surfaces are now nonpolar. It is interesting to note that the higher the hydroxylation level the lower the intensity of the interaction. We also find the same dependence with the Agcoordination number: a silver layer interacts (per surface atom) less than isolated atoms. Furthermore, when the surface is hydroxylated, the interactions are typically 1 order of magnitude less than covalent interactions, and comparable to those observed on quartz surface. This shows the importance of the hydroxylation for the silver-silica interaction, a result comparable with that obtained for the $\mathrm{Cu} / \mathrm{SiO}_{2}$ system. ${ }^{48}$ The dispersion contributions play an important role as can be seen from the DFT-D2 results (see Table 2). For one Ag layer, the 
adhesion energy is now $-0.12 \mathrm{eV} /$ at for the partially hydroxylated surface and $-0.15 \mathrm{eV} /$ at for the fully hydroxylated surface. These values slightly decrease for the 4-layers thick slab of silver $(-0.19$ and $-0.16 \mathrm{eV} / \mathrm{at})$. Note that the dependence with the Ag-coordination is weaker for the fully hydroxylated surface. The average value, $-0.16 \mathrm{eV} /$ at, corresponds to a weak adhesion energy without charge transfer (van der Waals like interaction), and has been used to determine the $\eta$ parameter of the silver-silica potential eq 1 ). We have found $\eta^{\text {crist }}=5.0$ (see Table 3. As can be seen, the dispersion contributions are

Table 3. $\eta$ Parameter for the $\mathrm{Ag}-\mathrm{SiO}_{2}$ Interaction Potential (Eq 1) Determined from the DFT-D2 Calculations of Silver Adhesion on Hydroxylated Cristobalite and from Experimental Wetting Angle Measurements on Amorphous Silica

$\begin{array}{ccc}\text { quartz } & \text { amorphous silica } & \text { hydroxylated cristobalite } \\ 1.0 & 2.5 & 5.0\end{array}$

dominant. It is thus important to keep in mind that these values are sensitive to the level of approximation, and that better methods can be used to improve the quality. ${ }^{59}$ A comparison with experiments is also fruitful, as proposed now in the case of amorphous silica.

3.3. Ag-Amorphous Silica Potential. We have performed Monte Carlo simulations of a liquid nanodroplet on flat amorphous silica surface. The droplet size, $3 \mathrm{~nm}$, is large enough to have a good precision on the mean value of the aspect ratio, which relates to the wetting angle through the relation (see also Figure 3):

$$
\frac{H}{D}=\frac{1-\cos \theta}{2}
$$

We have used the silver-silica potential eq 1 with $\varepsilon$ and $\sigma$ previously determined on quartz, and where the unknown parameter $\eta^{\text {amorph }}$ has been varied. This parameter modulates the intensity of the interaction, and thus has an impact on the wetting angle and the aspect ratio. The simulations have been performed at several temperatures between 1000 and $1300 \mathrm{~K}$, corresponding to the range of temperatures used in experiments. The results are given in Figure 4. As can be seen, the aspect ratio is strongly influenced by $\eta^{\text {amorph }}$, with a weak dependence with temperature. The experimental measurement by Eustathopoulos and co-workers ${ }^{44-46}\left(140^{\circ}\right.$, i.e., $\mathrm{H} / \mathrm{D}=$ 0.88 ) can be reproduced for $\eta^{\text {amorph }}=2.5$ (see Table 3$)$.

The value of $\eta^{\text {amorph }}$ is half that of $\eta^{\text {crist }}$. The difference cannot be attributed to surface hydroxylation or bulk density since they are similar for both substrates. It cannot either be explained by the fact that wetting angle measurements are done in the liquid phase, since experimental investigation done by vapor deposition of silver atoms on amorphous silica substrate indicate that the aspect ratio of solid nanoparticles at room temperature is approximately 0.83 for a mean size of $2.6 \pm 0.4$ $\mathrm{nm}$, similar to that obtained in the liquid phase. However, several differences between the substrates may be evoked to explain this factor. First, the bulk structure is not the same in both cases (crystal versus amorphous structure). One expects lower interaction with the amorphous silica compared with the crystal. Another explanation is the following: the DFT calculations are done on nanometric systems, while the wetting angle measurements are done on macroscopic systems. As a consequence, the latter might be sensitive to unfavorable

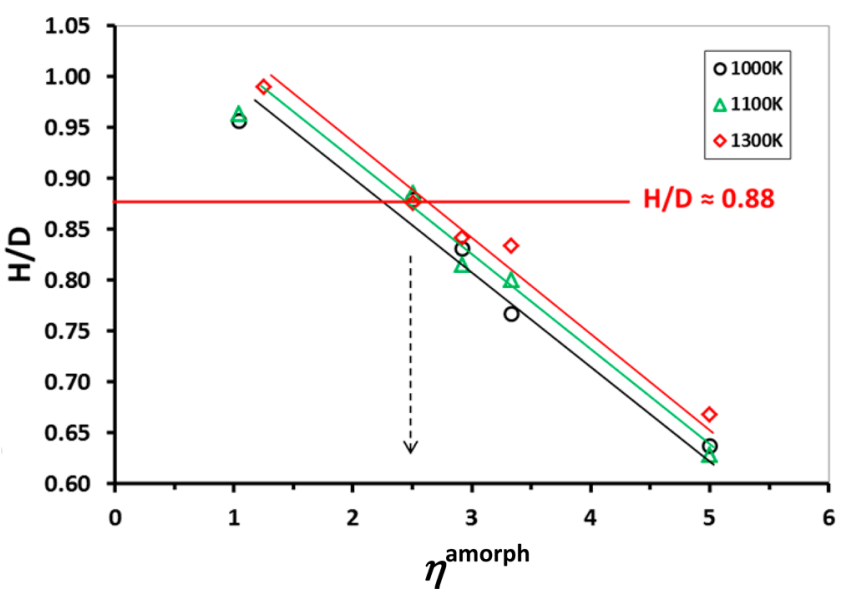

Figure 4. Equilibrium aspect ratio $\mathrm{H} / \mathrm{D}$ of a silver nanodroplet deposited on the amorphous silica support obtained by Monte Carlo simulation, for three temperatures, and as a function of the free parameter $\eta^{\text {amorph }}$ eq 1 . Horizontal red line: experimental determination by Eustathopoulos and co-workers. ${ }^{44-46}$

surface defects (geometric or chemical) even if their density is low. The discrepancy might also be an indication that the approximation level used to perform the calculations (DFTD2) is not accurate enough. Further improvement is considered.

3.4. Melting and Freezing of a Ag Nanoparticle on Amorphous Silica. As an illustration of the influence of the silica support on the deposited nanoparticle properties, we have first considered the melting of a $2 \mathrm{~nm}$ silver nanoparticle on the amorphous silica substrate using canonical Monte Carlo simulations. A calorimetric curve is determined by successive simulations performed at increasing temperatures for which an equilibrium state is achieved (typically $5 \times 10^{5}$ Monte Carlo steps per atom for each temperature). We have focused on the icosahedron with 309 atoms. The melting temperature is clearly identified on the calorimetric curve by the step in the average energy of the system (see Figure 5). The substrate effect is evidenced by comparing the free and supported nanoparticles. The melting temperature is around $720 \mathrm{~K}$ for the free nanoparticle and $700 \mathrm{~K}$ for the supported nanoparticle. This reduction of the melting temperature is at variance compared

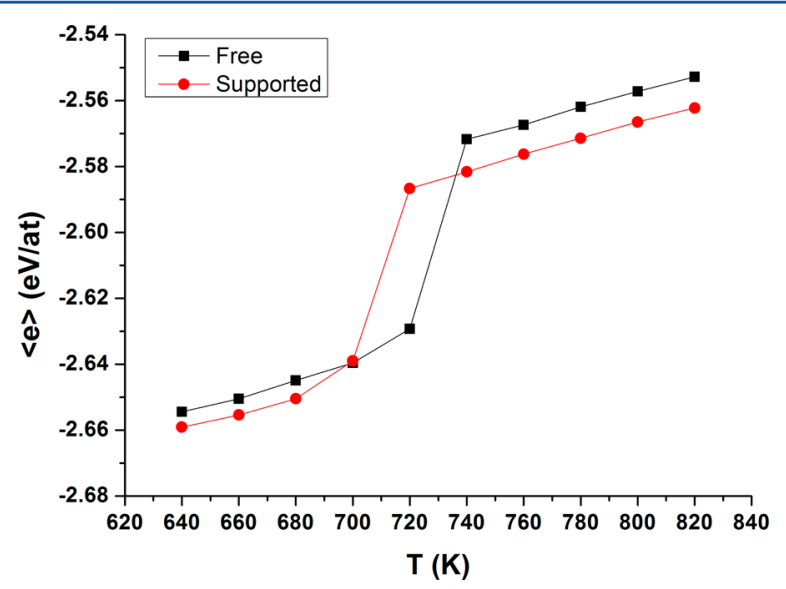

Figure 5. Melting calorimetric curve (energy per atom versus temperature) of a silver nanoparticle (Ih 309) obtained by Monte Carlo simulations in the canonical ensemble. Key: squares, free nanoparticle; circles, nanoparticle deposited on the silica support. 
with what is generally observed for supported nanoparticles. ${ }^{42}$ This is explained by the fact that the icosahedron structure is most probably metastable for a $2 \mathrm{~nm}$ sized silver nanoparticle at that temperature. At zero temperature, the stable structure is the Marks decahedron for a free nanoparticle, and the fcc truncated octahedron for a nanoparticle deposited on silica. ${ }^{60}$ The substrate thus destabilizes the icosahedron, and somehow favors the mobility of the atoms at the interface. This is confirmed by the examination of the atomic configurations (see Figure 6) showing that the melting of the supported nanoparticle occurs at the interface with the support.
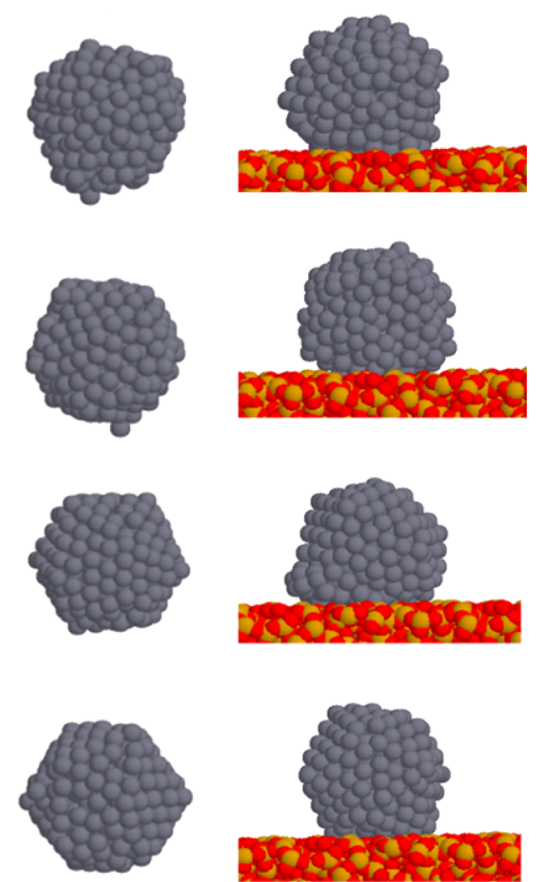

Figure 6. Configurations of a silver nanoparticle (Ih 309) as a function of the temperature (melting). Left: free nanoparticle. Right: nanoparticle deposited on silica. From bottom to top: 680, 700, 720, and $740 \mathrm{~K}$.

We have then examined the reverse process (freezing) for a nanodroplet of similar size, containing exactly 300 atoms in order to avoid any magic number which could favor a particular structure for the solid. The nanodroplet is first completely melted at $900 \mathrm{~K}$, well above the melting temperature, and progressively cooled down to $650 \mathrm{~K}$, where it is maintained until the system freezes. At that temperature $(50 \mathrm{~K}$ below the melting temperature), the system has enough mobility to allow equilibration as long as it remains in the metastable liquid state. When the freezing occurs, the structure is not systematically the same: among the five independent runs that we have performed to explore different realizations, we have observed the formation of an icosahedral structure in one case, and an fcc truncated octahedron in the four other cases (see Figure 7). The substrate clearly favors the formation of the fcc structure. Here again, we have evidenced the effect of the substrate since in the case of a small free nanodroplet the most favorable configurations are noncrystalline (icosahedron or decahedron structure). The presence of a flat $\mathrm{Ag} / \mathrm{SiO}_{2}$ interface most probably plays an important role in the nucleation of the crystal structure.
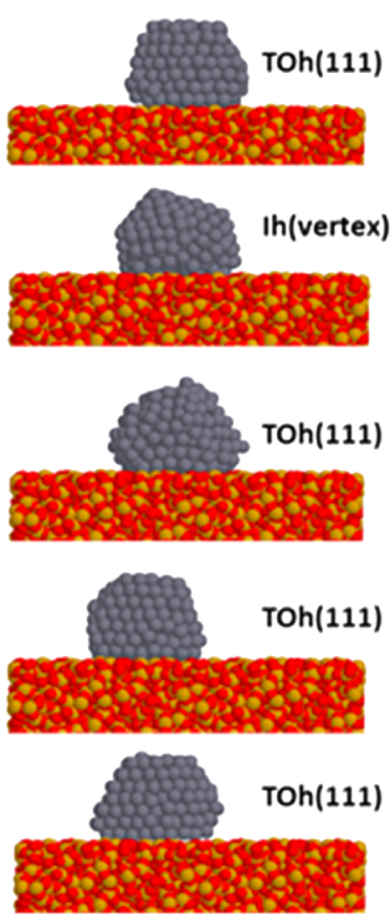

Figure 7. Configurations of a silver nanoparticle ( 300 atoms) obtained after freezing at $650 \mathrm{~K}$, for five independent runs.

\section{CONCLUSION}

This work presents DFT calculations of adsorption and adhesion energies of silver atoms or layers on cristobalite, a silica crystal. It is shown that the hydroxylation of the surface greatly influences the Ag-silica interaction. For hydroxyl surface density close to experimental observations in the conditions encountered in nanoparticle deposition, between 3.6 $\mathrm{OH} / \mathrm{nm}^{2}$ and $7.2 \mathrm{OH} / \mathrm{nm}^{2}$, the interaction is weak, van der Waals like. Our results are compared with that obtained by Vakula et al. ${ }^{47}$ on quartz, another silica crystal, the differences being imputed to the fact that the structure and density of the bulk and the surface of both substrates are significantly different and that we have taken into account the dispersion terms within the DFT-D2 approximation. We have also shown that the Ag-silica interaction barely decreases when the hydroxylation level of cristobalite increases. We have also considered the case of amorphous silica, and used wetting angle experiments to determine the intensity of the $\mathrm{Ag}$-silica interaction. All available data have been used to parametrize a Lennard-Jones potential (eq 1, Tables 1 and 3) to describe the silver-silica interaction for quartz, hydroxylated cristobalite and amorphous silica. Using Monte Carlo simulations in the canonical ensemble, we have shown the influence of the silica support on the morphology and structure of silver nanoparticles on silica obtained by recrystallization of silver nanodroplets.

\section{AUTHOR INFORMATION}

\section{Corresponding Author}

*(J.P.) E-mail: puibasset@cnrs-orleans.fr. Telephone: 33238 255803.

ORCID ${ }^{\circledR}$

J. Puibasset: 0000-0003-0286-3160

Notes

The authors declare no competing financial interest. 


\section{ACKNOWLEDGMENTS}

A.C.N. acknowledges a grant from the education and research ministry for his Ph.D. We are grateful for fruitful discussions with M. Benoit, and acknowledge C. Andreazza-Vignolle and P. Andreazza for stimulating discussions and for providing nonpublished data on silver nanoparticles deposited on amorphous silica. This work received computational support from the French HPC resources GENCI-IDRIS (Grant 2015096829).

\section{REFERENCES}

(1) Henry, C. R. Surface Studies of Supported Model Catalysts. Surf. Sci. Rep. 1998, 31, 231-325.

(2) Henry, C. R. Morphology of Supported Nanoparticles. Prog. Surf. Sci. 2005, 80, 92-116.

(3) Wallace, W. T.; Min, B. K.; Goodman, D. W. The Nucleation, Growth, and Stability of Oxide-Supported Metal Clusters. Top. Catal. 2005, 34, 17-30.

(4) Freund, H.-J. Model Systems in Heterogeneous Catalysis: Selectivity Studies at the Atomic Level. Top. Catal. 2008, 48, 137-144.

(5) Campbell, C. T. Ultrathin Metal Films and Particles on Oxide Surfaces: Structural, Electronic and Chemisorptive Properties. Surf. Sci. Rep. 1997, 27, 1-111.

(6) Renaud, G. Oxide Surfaces and Metal/Oxide Interfaces Studied by Grazing Incidence X-Ray Scattering. Surf. Sci. Rep. 1998, 32, 5-90.

(7) Pauwels, B.; Van Tendeloo, G.; Bouwen, W.; Theil Kuhn, L.; Lievens, P.; Lei, H.; Hou, M. Low-Energy-Deposited Au Clusters Investigated by High-Resolution Electron Microscopy and Molecular Dynamics Simulations. Phys. Rev. B: Condens. Matter Mater. Phys. 2000, 62, 10383-10393.

(8) Robach, O.; Renaud, G.; Barbier, A. Structure and Morphology of the $\mathrm{Ag} / \mathrm{MgO}(001)$ Interface During in Situ Growth at Room Temperature. Phys. Rev. B: Condens. Matter Mater. Phys. 1999, 60, 5858-5871.

(9) Graoui, H.; Giorgio, S.; Enry, C. R. Effect of the Interface Structure on the High-Temperature Morphology of Supported Metal Clusters. Philos. Mag. B 2001, 81, 1649-1658.

(10) Vervisch, W.; Mottet, C.; Goniakowski, J. Theoretical Study of the Atomic Structure of Pd Nanoclusters Deposited on a $\mathrm{MgO}(100)$ Surface. Phys. Rev. B: Condens. Matter Mater. Phys. 2002, 65, 245411.

(11) Goniakowski, J.; Mottet, C. Palladium Nano-Clusters on the $\mathrm{MgO}(100)$ Surface: Substrate-Induced Characteristics of Morphology and Atomic Structure. J. Cryst. Growth 2005, 275, 29-38.

(12) Mottet, C.; Goniakowski, J. Influence of Epitaxial Strain on Supported Metal Cluster Shapes Via Atomistic Simulations. J. Comput. Theor. Nanosci. 2007, 4, 326-334.

(13) Jelea, A.; Mottet, C.; Goniakowski, J.; Rossi, G.; Ferrando, R. Canonical Molecular Dynamics Simulations for Crystallization of Metallic Nanodroplets on $\mathrm{MgO}(100)$. Phys. Rev. B: Condens. Matter Mater. Phys. 2009, 79, 165438.

(14) Ferrando, R.; Rossi, G.; Levi, A. C.; Kuntová, Z.; Nita, F.; Jelea, A.; Mottet, C.; Barcaro, G.; Fortunelli, A.; Goniakowski, J. Structures of Metal Nanoparticles Adsorbed on $\mathrm{MgO}(001)$. I. Ag and Au. J. Chem. Phys. 2009, 130, 174702.

(15) Barcaro, G.; Sementa, L.; Negreiros, F. R.; Ferrando, R.; Fortunelli, A. Interface Effects on the Magnetism of Copt-Supported Nanostructures. Nano Lett. 2011, 11, 5542-5547.

(16) Ferrando, R; Barcaro, G.; Fortunelli, A. Structures of Small Au Clusters on $\mathrm{MgO}(001)$ Studied by Density-Functional Calculations. Phys. Rev. B: Condens. Matter Mater. Phys. 2011, 83, 045418.

(17) Paz-Borbón, L. O.; Barcaro, G.; Fortunelli, A.; Levchenko, S. V. $\mathrm{Au}_{\mathrm{n}}$ Clusters $(\mathrm{N}=1-6)$ Supported on $\mathrm{MgO}(100)$ Surfaces: Effect of Exact Exchange and Dispersion Interactions on Adhesion Energies. Phys. Rev. B: Condens. Matter Mater. Phys. 2012, 85, 155409.

(18) Stankic, S.; Cortes-Huerto, R.; Crivat, N.; Demaille, D.; Goniakowski, J.; Jupille, J. Equilibrium Shapes of Supported Silver Clusters. Nanoscale 2013, 5, 2448-2453.
(19) Atanasov, I.; Barcaro, G.; Negreiros, F. R.; Fortunelli, A.; Johnston, R. L. Modelling the Metal-on-Top Effect for Pd Clusters on the $\mathrm{MgO}\{100\}$ Substrate. J. Chem. Phys. 2013, 138, 224703.

(20) Penuelas, J.; Andreazza, P.; Andreazza-Vignolle, C.; Tolentino, H. C. N.; De Santis, M.; Mottet, C. Controlling Structure and Morphology of Copt Nanoparticles through Dynamical or Static Coalescence Effects. Phys. Rev. Lett. 2008, 100, 115502.

(21) Penuelas, J.; Andreazza-Vignolle, C.; Andreazza, P.; Ouerghi, A.; Bouet, N. Temperature Effect on the Ordering and Morphology of Copt Nanoparticles. Surf. Sci. 2008, 602, 545-551.

(22) Iler, R. K. The Chemistry of Silica; John Wiley \& Sons: New York, 1979.

(23) Zhuravlev, L. T. The Surface Chemistry of Amorphous Silica. Zhuravlev Model. Colloids Surf., A 2000, 173, 1-38.

(24) Ewing, C. S.; Bhavsar, S.; Veser, G.; McCarthy, J. J.; Johnson, J. K. Accurate Amourphous Silica Surface Models from First-Principles Thermodynamics of Surface Dehydroxylation. Langmuir 2014, 30, 5133-5141.

(25) Tsuneyuki, S.; Tsukada, M.; Aoki, H.; Matsui, Y. First Principles Interatomic Potential of Silica Applied to Molecular Dynamics. Phys. Rev. Lett. 1988, 61, 869-872.

(26) van Beest, B. W. H.; Kramer, G. J.; van Santen, R. A. Force Fields for Silicas and Aluminophosphates Based on $A b$ Initio Calculations. Phys. Rev. Lett. 1990, 64, 1955-1958.

(27) Guissani, Y.; Guillot, B. A Numerical Investigation of the LiquidVapor Coexistence Curve of Silica. J. Chem. Phys. 1996, 104, 76337644.

(28) Cruz-Chu, E.; Aksimentiev, A.; Schulten, K. Water-Silica Force Field for Simulating Nanodevices. J. Phys. Chem. B 2006, 110, 2149721508.

(29) Allan, D. C.; Teter, M. P. Nonlocal Pseudopotentials in Molecular-Dynamical Density-Functional Theory: Application to $\mathrm{SiO}_{2}$. Phys. Rev. Lett. 1987, 59, 1136-1139.

(30) Lee, C.; Gonze, X. Lattice Dynamics and Dielectric Properties of $\mathrm{SiO}_{2}$ Stishovite. Phys. Rev. Lett. 1994, 72, 1686-1689.

(31) Liu, F.; Garofalini, S. H.; King-Smith, R. D.; Vanderbilt, D. FirstPrinciples Studies on Structural Properties of Beta-Cristobalite. Phys. Rev. Lett. 1993, 70, 2750-2753.

(32) Rozanska, X.; Delbecq, F.; Sautet, P. Reconstruction and Stability of $\beta$-Cristobalite 001, 101, and 111 Surfaces During Dehydroxylation. Phys. Chem. Chem. Phys. 2010, 12, 14930-14940.

(33) Benoit, M.; Ispas, S.; Jund, P.; Jullien, R. Model of Silica Glass from Combined Classical and $A b$ Initio Molecular-Dynamics Simulations. Eur. Phys. J. B 2000, 13, 631-636.

(34) Van Ginhoven, R. M.; Jónsson, H.; Corrales, L. R. Silica Glass Structure Generation for $A b$ Initio Calculations Using Small Samples of Amorphous Silica. Phys. Rev. B: Condens. Matter Mater. Phys. 2005, 71, 024208 .

(35) Tielens, F.; Gervais, C.; Lambert, J. F.; Mauri, F.; Costa, D. Ab Initio Study of the Hydroxylated Surface of Amorphous Silica: A Representative Model. Chem. Mater. 2008, 20, 3336-3344.

(36) Ewing, C. S.; Veser, G.; McCarthy, J. J.; Johnson, J. K.; Lambrecht, D. S. Effect of Support Preparation and Nanoparticle Size on Catalyst-Support Interactions between Pt and Amorphous Silica. J. Phys. Chem. C 2015, 119, 19934-19940.

(37) Sherry, L. J.; Chang, S.-H.; Schatz, G. C.; Van Duyne, R. P.; Wiley, B. J.; Xia, Y. Localized Surface Plasmon Resonance Spectroscopy of Single Silver Nanocubes. Nano Lett. 2005, 5, 2034-2038.

(38) Atwater, H. A.; Polman, A. Plasmonics for Improved Photovoltaic Devices. Nat. Mater. 2010, 9, 205-213.

(39) Seney, C. S.; Gutzman, B. M.; Goddard, R. H. Correlation of Size and Surface-Enhanced Raman Scattering Activity of Optical and Spectroscopic Properties for Silver Nanoparticles. J. Phys. Chem. C 2009, 113, 74-80.

(40) Haes, A. J.; Zou, S.; Schatz, G. C.; Van Duyne, R. P. A Nanoscale Optical Biosensor: The Long Range Distance Dependence of the Localized Surface Plasmon Resonance of Noble Metal Nanoparticles. J. Phys. Chem. B 2004, 108, 109-116. 
(41) Rosato, V.; Guillope, M.; Legrand, B. Thermodynamical and Structural Properties of F.C.C. Transition Metals Using a Simple Tight-Binding Model. Philos. Mag. A 1989, 59, 321-336.

(42) Mottet, C.; Goniakowski, J.; Baletto, F.; Ferrando, R.; Treglia, G. Modeling Free and Supported Metallic Nanoclusters: Structure and Dynamics. Phase Transitions 2004, 77, 101-113.

(43) Young, V. Y.; Gibbs, R. A.; Winograd, N.; Kim, K. S. X-Ray Photoemission Studies of Atom Implanted Solids: $\mathrm{Ag}$ and $\mathrm{Au}$ in $\mathrm{SiO}_{2}$. Chem. Phys. Lett. 1978, 54, 378-382.

(44) Eustathopoulos, N.; Drevet, B. Interfacial Bonding, Wettability and Reactivity in Metal/Oxide Systems. J. Phys. III 1994, 4, 18651881.

(45) Eustathopoulos, N.; Drevet, B. Determination of the Nature of Metal-Oxide Interfacial Interactions from Sessile Drop Data. Mater. Sci. Eng., A 1998, 249, 176-183.

(46) Sangiorgi, R.; Muolo, M. L.; Chatain, D.; Eustathopoulos, N. Wettability and Work of Adhesion of Nonreactive Liquid Metals on Silica. J. Am. Ceram. Soc. 1988, 71, 742-748.

(47) Vakula, N. I.; Kuramshina, G. M.; Pentin, Y. A. Molecular Simulation of Interactions between Silver Clusters and an $\alpha$-Quartz Surface. Russ. J. Phys. Chem. A 2013, 87, 296-302.

(48) Nagao, K.; Neaton, J. B.; Ashcroft, N. W. First-Principles Study of Adhesion at $\mathrm{Cu} / \mathrm{SiO}_{2}$ Interfaces. Phys. Rev. B: Condens. Matter Mater. Phys. 2003, 68, 125403.

(49) Allen, M. P.; Tildesley, D. J. Computer Simulation of Liquids; Clarendon Press: Oxford, U.K., 1987.

(50) Kresse, G.; Hafner, J. Ab Initio Molecular Dynamics for Liquid Metals. Phys. Rev. B: Condens. Matter Mater. Phys. 1993, 47, 558-561.

(51) Kresse, G.; Joubert, D. From Ultrasoft Pseudopotentials to the Projector Augmented-Wave Method. Phys. Rev. B: Condens. Matter Mater. Phys. 1999, 59, 1758-1775.

(52) Perdew, J. P.; Burke, K.; Ernzerhof, M. Generalized Gradient Approximation Made Simple. Phys. Rev. Lett. 1996, 77, 3865-3868.

(53) Grimme, S. Semiempirical Gga-Type Density Functional Constructed With a Long-Range Dispersion Correction. J. Comput. Chem. 2006, 27, 1787-1799.

(54) Fang, H.; Dove, M. T.; Refson, K. Ag-Ag Dispersive Interaction and Physical Properties of $\mathrm{Ag}_{3} \mathrm{Co}(\mathrm{CN})_{6}$. Phys. Rev. B: Condens. Matter Mater. Phys. 2014, 90, 054302.

(55) Chuang, I. S.; Maciel, G. E. A Detailed Model of Local Structure and Silanol Hydrogen Bonding of Silica Gel Surfaces. J. Phys. Chem. B 1997, 101, 3052-3064.

(56) Puibasset, J.; Pellenq, R. J.-M. Water Adsorption on Hydrophilic Mesoporous and Plane Silica Substrates: A Grand Canonical Monte Carlo Simulation Study. J. Chem. Phys. 2003, 118, 5613-5622.

(57) Puibasset, J.; Pellenq, R. J.-M. Grand Canonical Monte Carlo Simulation Study of Water Structure on Hydrophilic Mesoporous and Plane Silica Substrates. J. Chem. Phys. 2003, 119, 9226-9232.

(58) Goniakowski, J.; Finocchi, F.; Noguera, C. Polarity of Oxide Surfaces and Nanostructures. Rep. Prog. Phys. 2008, 71, 016501.

(59) Klimeš, J.; Michaelides, A. Perspective: Advances and Challenges in Treating Van Der Waals Dispersion Forces in Density Functional Theory. J. Chem. Phys. 2012, 137, 120901.

(60) Ngandjong, A. C.; Mottet, C.; Puibasset, J. Influence of the Silica Support on the Structure and the Morphology of Silver Nanoparticles: A Molecular Simulation Study. J. Phys. Chem. C 2016, 120, 8323-8332. 